\title{
Correlated evolution of personality, morphology, and performance
}

Elizabeth M.A. Kern ${ }^{\dagger a}$, Detric Robinson ${ }^{\dagger \mathrm{a}}$, Erika Gass ${ }^{\mathrm{a}}$, John Godwin ${ }^{\mathrm{a}}$, and R. Brian

$4 \quad$ Langerhans $^{\mathrm{a}}$

a Department of Biological Sciences and W.M. Keck Center for Behavioral Biology, North Carolina State University, Raleigh, NC, USA

*Correspondence: E. Kern. Present address: Department of Life Science, Ewha Womans

11 E-mail: ekern@ewha.ac.kr

Phone: +82-02-3277-4038

${ }^{\dagger}$ These authors contributed equally to the paper and their names are given in alphabetical order.

Word count: 5,040 


\section{Abstract}

17 Evolutionary change in one trait can elicit evolutionary changes in other traits due to genetic correlations. This constrains the independent evolution of traits and can lead to unpredicted

19 ecological and evolutionary outcomes. Animals might frequently exhibit genetic associations 20 among behavioral and morphological-physiological traits, because the physiological mechanisms

21 behind animal personality can have broad multi-trait effects and because many selective agents

22 influence the evolution of multiple types of traits. However, we currently know little about 23 genetic correlations between animal personalities and non-behavioral traits. We tested for 24 associations between personality, morphology, and locomotor performance by comparing 25 zebrafish (Danio rerio) collected from the wild and then selectively bred for either a proactive or 26 reactive stress coping style ("bold" or "shy" phenotypes). Based on adaptive hypotheses of 27 correlational selection in the wild, we predicted that artificial selection for boldness would 28 produce correlated evolutionary responses of larger caudal regions and higher fast-start escape 29 performance (and the opposite for shyness). After 4-7 generations, morphology and locomotor 30 performance differed between personality lines: bold zebrafish exhibited a larger caudal region 31 and higher fast-start performance than fish in the shy line, matching predictions. Individual-level 32 phenotypic correlations suggested that pleiotropy or physical gene linkage likely explained the 33 correlated response of locomotor performance, while the correlated response of body shape may

34 have reflected linkage disequilibrium, which is breaking down each generation in the lab. Our 35 results indicate that evolution of personality can result in concomitant changes in morphology 36 and whole-organism performance, and vice versa.

Keywords: Behavioural syndromes, morphology, personality, pleiotropy, stress coping style, swimming performance, zebrafish 


\section{Introduction}

Evolutionary response to selection depends not only on the strength and nature of selection, but also on the heritability of the trait in question and its genetic correlations with other traits (Agrawal \& Stinchcombe, 2009; Falconer \& MacKay, 1996; Lande, 1979). Because genetic correlations are common, selection on one trait often affects the evolution of other traits (Brodie, 1989; Ketterson, Atwell, \& McGlothlin, 2009; Pigliucci \& Preston, 2004). Understanding how and why this happens has received considerable attention in animal personalities, where behavioral traits covary to produce consistently distinct "personalities," "temperaments," or "behavioral syndromes” (Réale, Dingemanse, Kazem, \& Wright, 2010; Réale, Reader, Sol, McDougall, \& Dingemanse, 2007; Sih, Bell, \& Johnson, 2004). However, we know little about whether animal personalities have genetic associations with non-behavioral traits, even though such associations should be expected and could have major ecological and evolutionary implications (Sih, Cote, Evans, Fogarty, \& Pruitt, 2012; Wolf \& Weissing, 2012). We suggest that animal personalities may often exhibit genetic correlations (i.e., heritable nonrandom associations among traits) with seemingly disparate non-behavioral traits due to (1) correlational selection on behavioral and non-behavioral traits or (2) pleiotropic effects of the genes and physiological mechanisms that underlie animal personalities.

First, correlational selection may often occur in nature since selective forces can influence both behavioral traits and non-behavioral traits, such as morphology and physiology (Dewitt, Sih, \& Hucko, 1999; Endler, 1995; Ketterson et al., 2009; Schluter, 2010; Sinervo \&

63 Svensson, 2002; Vervust, Grbac, \& Van Damme, 2007). Correlational selection describes cases

64 where the fitness effect of one trait depends on the value of another trait. For instance, certain

65 behaviors may produce high fitness only when combined with specific morphologies. This

66 frequently produces genetic correlations (Cheverud, 1984; Jones, Arnold, \& Bürger, 2003; Lande

67 \& Arnold, 1983; Lynch \& Walsh, 1998; McGlothlin, Parker, Nolan, \& Ketterson, 2005; Phillips

68 \& Arnold, 1989; Roff \& Fairbairn, 2012; Sinervo \& Svensson, 2002), as in garter snakes, where

69 correlational selection on color pattern and predator-escape behavior results in covariance among 70 these traits (Brodie, 1992).

Correlational selection on personality traits and non-behavioral traits may be more

72 common than we realize, since certain behaviors should vary in fitness depending on other traits.

73 For instance, risk-prone, aggressive individuals may require great strength, speed, or large size to 
74 achieve high fitness. Correlational selection can produce genetic correlations through pleiotropy

75 (where genes affect multiple traits), genetic linkage (where genes are located nearby on a

76 chromosome), or linkage disequilibrium (where separate traits exhibit associations due to

77 correlational selection or non-random mating; Falconer \& MacKay, 1996; Lynch \& Walsh,

78 1998). Regardless of the source of genetic correlations, understanding the existence and strength

79 of these associations is important for understanding adaptation. In reality, traits do not adapt to

80 environments independently — rather, selection acts on whole-organism phenotypes, resulting in

81 organisms with evolved adaptations that reflect integrated suites of traits (Ghalambor, Walker, \&

82 Reznick, 2003; Murren, 2012; Pigliucci \& Preston, 2004; Réale, Garant, et al., 2010; Santos \&

83 Cannatella, 2011).

84 Second, irrespective of correlational selection, prior work suggests that genes responsible

85 for animal personalities may have widespread pleiotropic effects. The physiological mechanisms

86 underlying animal personalities often pleiotropically affect traits such as dispersal behavior,

87 metabolic rate, immune capacity, lifespan, age at reproduction, and growth rate (Biro \& Stamps,

88 2010; Careau, Réale, Humphries, \& Thomas, 2010; Réale, Garant, et al., 2010). These same

89 factors could also affect other traits like morphology or whole-organism performance abilities

90 (Bourdeau \& Johansson, 2012; Dickey, McCarley, \& Shenton, 2002; Johansson \& Andersson,

91 2009; Selman, Lumsden, Bünger, Hill, \& Speakman, 2001; Swallow \& Hayes, 2009), yet few

92 studies have examined whether animal personalities exhibit genetic associations with

93 morphological-physiological traits. Considering what we know about hormone-mediated suites

94 of traits (Adkins-Regan, 2005; McGlothlin \& Ketterson, 2008), and given the diverse sets of trait

95 correlations involved in pace-of-life syndromes (Careau et al., 2010; Réale, Garant, et al., 2010),

96 we should expect to find a range of associations between animal personalities and

97 morphological-physiological traits owing to shared genetic or physiological bases. Indeed,

98 artificial selection on behavior, such as during domestication, can result in changes in seemingly

99 disparate traits such as color, skull shape, and seasonal reproductive patterns (Trut, Oskina, \&

100 Kharlamova, 2009; Trut, Plyusnina, \& Oskina, 2004). Identifying such associations will help us

101 understand the evolution of complex phenotypes and the limitations to adaptive evolution (since

102 trait correlations can present trade-offs that bias the direction of evolution; Pigliucci \& Preston,

103 2004; Pruitt \& Riechert, 2012; Schluter, 1996). Here we use zebrafish to provide one of the first 
104 tests of the notion that animal personalities might exhibit genetic associations with

105 morphological and performance traits.

Three general types of traits-behavior (animal personality), morphology (body shape),

107 and locomotor ability (fast-start swimming performance) — could frequently show genetic

108 correlation for a number of non-mutually exclusive reasons. First, changes in behavior,

109 metabolism, or hormones might induce changes in morphology (Bourdeau \& Johansson, 2012;

110 Johansson \& Andersson, 2009). Secondly, morphological changes should affect fast-start

111 locomotor performance via trait codependence (sensu Dewitt et al., 1999), because swimming

112 ability partially derives from the thrust generated by the caudal region of a fish (the two traits are

113 mechanically linked). Further, correlational selection might favor particular trait combinations

114 such as (1) trait complementation, where boldness enhances foraging or mating only when

115 combined with high fast-start performance, (2) trait cospecialization, where bold, fast individuals

116 and shy, slow individuals have high fitness because their trait combinations influence different

117 fitness components (e.g., the former may have higher mating success but low longevity, while

118 the latter may have lower mating success but high longevity), or (3) trait compensation, where

119 bold individuals encounter more predatory strikes but compensate with defensive morphologies

120 or rapid locomotor escape abilities. Any combination of these underlying causes could lead to

121 the evolution of genetic correlations among personality, morphology, and performance (Wolf \&

122 Werner, 1994).

123 We investigated whether these three traits evolve independently or in concert using

124 artificial selection with zebrafish (Danio rerio). We compared lines selected for bold or shy

125 behavior to determine whether body morphology or locomotor performance exhibited correlated

126 responses to behavioral selection. If genetic correlations exist between animal personalities and

127 these non-behavioral traits, then body morphology and swimming ability should diverge between

128 selection lines and appear as a correlated response to artificial selection (Carere \& van Oers,

129 2004; Houde, 1994; Wilkinson \& Reillo, 1994). We specifically predicted that artificial selection

130 for boldness would elicit correlated evolutionary responses of larger caudal regions and higher

131 fast-start escape performance (and the reverse for shyness).

132 While zebrafish are a well-established model laboratory organism (Norton \& Bally-Cuif,

133 2010; Ribas \& Piferrer, 2014; Ruzicka et al., 2015), less is known about their ecology in the

134 wild. Zebrafish tend to inhabit shallow, slow-moving freshwater where they use the entire water 
135 column and occupy both vegetated margins and open areas (Spence et al., 2006). They respond

136 strongly to predator cues (Dill, 1974), and their antipredator behavior is heritable and tends to

137 diminish in lab strains (Robison \& Rowland, 2005; Wright, Nakamichi, Krause, \& Butlin, 2006).

138 Zebrafish face predation threats from multiple sources in the wild (Engeszer, Patterson, Rao, \&

139 Parichy, 2007), and appear to represent a likely candidate for experiencing correlational selection

140 on behaviors and non-behavioral traits in nature.

\section{Materials and Methods}

Wild zebrafish from Gaighata, India were selectively bred in captivity for bold or shy

144 personalities on the basis of stationary behavior in a stressful environment (see Wong et al., 2012

145 for a complete description of the experiment). Briefly, during an open field test zebrafish were

146 introduced to a novel arena for five minutes, and the amount of time they spent stationary was

147 recorded. Fish that exhibited at most 50 seconds of stationary behavior were bred together to

148 generate a bold line, and fish that exhibited at least 200 seconds of stationary behavior were bred

149 together to generate a shy line. The selective breeding program began with F1 fish, and selection

150 was repeated each generation. By the third generation, these two selection lines differed

151 consistently not only in stationary time but also in six different measures of stress and anxiety-

152 related behaviors (Wong et al., 2012). These sets of consistent differences in multiple behavioral

153 stress responses are variously termed either proactive and reactive stress coping styles, or bold

154 and shy behavioral phenotypes (Koolhaas, de Boer, Coppens, \& Buwalda, 2010; Øverli et al., 155 2007; Wong et al., 2012).

156 We examined body morphology and locomotor escape performance of adult zebrafish

157 from bold and shy lines in the fourth $(n=29)$, fifth $(n=29)$, and seventh $(n=67)$ generations

$158 \quad\left(n_{\text {total }}=125\right.$; Table S1). Zebrafish from each generation were age-matched across bold and shy

159 lines and were similar in body size (mean \pm standard error for standard length: bold females

$16028.32 \pm 0.75 \mathrm{~mm}$, shy females $27.23 \pm 0.65 \mathrm{~mm}$, bold males $26.62 \pm 0.53 \mathrm{~mm}$, shy males $26.52 \pm$ $1610.54)$.

162 In the fourth and fifth generations, fish were not tracked individually across open field 163 assays and measures of morphology and swimming performance. In the seventh generation, fish 164 were tracked individually across all experimental components, permitting individual-level 
165 correlation analyses among stationary behavior, body shape, and fast-start performance. All fish

166 were reared in a recirculating system at North Carolina State University on a 14:10 light:dark

167 cycle at $27.4^{\circ} \mathrm{C}$ and fed dry flakes ad libitum.

168

\section{Morphology}

We used geometric morphometrics to measure the body morphology of all fish. We digitized ten anatomical landmarks on lateral photographs of live individuals (Fig. 1a) using

172 tpsDig2 (Rohlf, 2013). Landmarks were located on the anterior tip of the snout on the upper jaw, 173 the back of the head (posterior aspect of the neurocranium), the anterior and posterior insertions 174 of the dorsal fin, the anterior attachments of the dorsal and ventral insertions of the caudal fin, 175 the anterior insertions of the anal and pelvic fins, the insertion of the operculum on the ventral 176 lateral profile, and the center of the eye. We generated shape variables (relative warps) using 177 tpsRelw (Rohlf, 2010). Prior to Generalized Procrustes Analysis in tpsRelw, we used tpsUtil 178 (Rohlf, 2012) to perform the unbend function, which removes postural differences. Landmarks 179 for unbending were located in the center of the eye, the posterior midpoint of the operculum, the center of the body between landmarks 3 and 7, and the base of the caudal fin between landmarks 5 and 6. Except for the eye landmark, these points were excluded from shape analyses because

182 they are not anatomically homologous. We used centroid size (the square root of the summed, 183 squared distances of all landmarks from their centroid) as the estimate for body size.

For analysis we retained 11 relative warps, explaining $95.5 \%$ of the shape variance. We tested for morphological differences between bold and shy lines using multivariate analysis of covariance (MANCOVA), with 11 shape variables (relative warps) as dependent variables,

187 coping style line, sex, and the interaction of sex and coping style line as independent variables, 188 and generation and centroid size as covariates (the latter controls for multivariate allometry). We initially included all possible interactions, and excluded non-significant interaction terms in the 190 final analysis.

191 To estimate the overall magnitude of shape differences between lines, we calculated 192 Procrustes distance between line means for each sex using tpsSmall (Rohlf, 2003). Procrustes 193 distance represents the standard metric of shape differences in geometric morphometrics (e.g., 194 Bookstein, 1996), and is closely approximated by Euclidean distance between landmarks after 195 Generalized Procrustes Analysis (Zelditch, Swiderski, \& Sheets, 2012). To evaluate the nature of 
the shape differences between lines, we calculated a divergence vector $(\mathbf{d})$ following Langerhans (2009b) and visualized this axis using thin-plate spline transformations. This divergence vector or "shape index" represents a canonical analysis of the coping style term from the MANCOVA and describes the linear combination of shape variables that exhibits the greatest differences between groups, adjusting for other factors in the model, in Euclidean space.

\section{Performance}

Swimming-performance trials took place in a square arena $(25.4 \mathrm{~cm}$ long $\times 25.4 \mathrm{~cm}$ wide $\times 6 \mathrm{~cm}$ deep) with a transparent Plexiglas base and opaque, black sides. Trials were recorded from below using a digital high-speed video camera (Model N4, Integrated Design Tools, Tallahassee, FL, USA) at $600 \mathrm{fps}$ and $1016 \times 1016$ pixel resolution. For the seventh generation of zebrafish, we recorded videos at $400 \mathrm{fps}$. Tests were performed after fish were at least seven months old. Testing order was randomized by individual (fourth and seventh generations) or systematically alternated between bold and shy lines (fifth generation). Water temperature was held constant $\left(27.4{ }^{\circ} \mathrm{C}\right)$ for all trials. We changed the water after each trial to avoid accumulating any alarm cues.

After placing an individual in the arena and allowing it to come to a standstill, we startled each fish by waving a hand over the tank and recorded the fast-start response. In fish, a fast-start is a brief, stereotyped burst of acceleration that enhances survival during predator encounters (Langerhans, 2009a, 2010). We recorded 2-4 responses per fish and selected one for analysis based on a qualitative assessment of the maximal response, as we wished to estimate maximum fast-start capacity and avoid inclusion of trials where individuals performed at less than their maximal capabilities (Losos, Creer, \& Schulte II, 2002).

We measured fish displacement during the first $\sim 70 \mathrm{~ms}$ of the fast-start response by digitizing the center of mass in each video frame using tpsDig. Center of mass was estimated for each individual based on the point along the center longitudinal line of the body (viewed from below) that showed the greatest latency for displacement in space as the fish's body bent during initiation of the C-start (Tytell \& Lauder, 2008). We smoothed displacement data using the mean-squared error quantic spline (Jeffrey A Walker, 1998), and used the smoothed data to calculate maximum velocity, average velocity, maximum acceleration, and average acceleration. We measured turning angle and mean angular velocity during stage one of the fast-start by 
227 digitizing the center of mass and the tip of the snout during the earliest part of the escape 228 response, in which the fish's body bends into a tightly curved " $\mathrm{C}$ " before moving forward (this was typically accomplished within the first $12 \mathrm{~ms}$ of the response).

In this way we obtained six performance variables from each video sequence: maximum velocity, average velocity, maximum acceleration, average acceleration, turning angle, and mean

232 angular velocity during stage one of the fast-start. We conducted principal components analysis

233 (PCA) on these six performance variables using the correlation matrix in order to reduce data

234 dimensionality, and retained only those PCs that explained more variance than expected by

235 broken stick criterion (three PCs). PC 1 explained over 40\% of the variance and captured a major

236 axis ranging from high-performance fish (high values for maximum velocity, maximum

237 acceleration, average velocity, and rotational velocity) to low-performance fish (low values for

238 those four variables). PC 2 described variation in turning angle, and PC 3 captured some of the

239 variation in average acceleration (Table 1).

240 We tested for differences in fast-start performance between phenotype lines by running

241 three general linear models, with each PC as the dependent variable and with coping style line,

242 sex, and their interaction as independent variables, and centroid size and generation as

243 covariates. We originally tested for all other possible interactions, but excluded them due to non-

244 significance. To provide an assessment of the magnitude of differences between coping style

245 lines, we report standardized effect sizes (Cohen's $d$, describing the difference between means in 246 standard deviation units; Cohen, 1988).

247 Finally, to examine how the three traits of personality, morphology, and performance 248 might be correlated among individuals raised in a common environment, we conducted two sets 249 of analyses using fish from the seventh lab-raised generation. First, we performed a general 250 linear model with stationary time as the dependent variable and the following as explanatory 251 variables: shape index, the three performance PCs, centroid size, and sex. We excluded all non252 significant terms. This model tested for direct associations between stationary behavior and 253 either morphology or performance or both, while controlling (i.e., adjusting) for potentially 254 confounding variables. That is, this analysis tests for behavior-morphology and behavior255 performance associations that are independent of any other measured variable (simple correlation 256 if only one term is included; partial correlations if multiple terms are included). Second, we 257 performed three separate general linear models, each model using one performance PC as the 
258 dependent variable and shape index, centroid size, and sex as explanatory variables. We again

259 excluded all non-significant terms. These analyses tested for direct associations between

260 performance and morphology, while again adjusting for possible confounding variables. That is,

261 these analyses test for morphology-performance associations via simple correlation or partial

262 correlation.

\section{Ethical Note}

The experiment was approved by the North Carolina State University IACUC (protocol 14-029-0). Fish were maintained in standard aquarium conditions and appeared to recover quickly after handling. No adverse effects on the overall health of the fish as a result of testing were apparent. The animals were later humanely euthanized in accordance with the approved animal care and use protocol (an overdose of tricaine methyl sulfonate).

\section{Results}

Body morphology showed statistically significant differences between bold and shy

274 zebrafish lines (Table 2). Average Procrustes distance between bold and shy lines was 0.017 for

275 females and 0.009 for males. Fish from the bold line exhibited a more elongate body with larger 276 caudal regions and shallower mid-bodies than fish from the shy line (Figure 1). The shape index

277 (visualized using thin-plate spline transformations in Figure 1), as well as Procrustes distances, 278 suggested that females showed a greater magnitude of morphological difference between bold 279 and shy coping styles than males (Figure 2), although we cannot reject the null hypothesis of no 280 sex-dependent differences (i.e., non-significant interaction term between sex and coping style). 281 Not surprisingly, we uncovered strong effects of allometry and sexual dimorphism on body 282 shape (Table 2). There were also significant effects of generation; the fifth generation had a 283 relatively longer body with a shallower mid-section, and the seventh generation had a longer 284 anal-fin insertion.

285 Swimming performance also showed significant differences between bold and shy lines 286 (Table 3; Table S2). Based on standardized effect sizes, females exhibited greater performance 287 differences between lines than males (PC 1: 0.91 vs. 0.23, PC 2: 0.36 vs. 0.27, PC 3: 0.61 vs. 
0.27). The bold line had higher fast-start performance (captured by PC 1) than the shy line, and

289 this effect was stronger in females than males (Figure 2). There was also a negative relationship

290 between centroid size and performance (smaller fish had higher PC 1 scores). Generation 4 had

291 highest performance and 5 had lowest. In PC 2, there was only one suggestive trend

292 (nonsignificant), where bold females tended to have higher turning angles than the shy line,

293 while males showed the opposite pattern. Bold females had higher PC 3 scores (average

294 acceleration) than shy females, while males again showed the opposite trend. Generation 4 had

295 lower PC 3 scores than the other two generations.

296 By explicitly testing direct associations between stationary time, morphology, and

297 performance, we found that behavior was directly correlated only with performance, not

298 morphology or other potentially confounding variables. Thus, we did not find behavior-

299 morphology associations when statistically controlling for performance. Performance PC 1

300 exhibited a significant association with stationary time $(r=-0.29, p=0.0194)$; the other two PCs

301 did not. Only shape index was significantly associated with performance PC $1(r=0.52, p<$

302 0.0001), indicating that fish with more elongate bodies and larger caudal regions exhibited

303 higher fast-start performance. No model terms were significantly related to the other PCs. Based

304 on these findings, we tested for an indirect association between stationary behavior and body

305 shape to quantify the potential strength of association owing to their shared relationships with

306 performance PC 1. Specifically, we calculated an indirect correlation coefficient as the product

307 of the two simple correlation coefficients (correlation between PC 1 and stationary time $x$

308 correlation between PC 1 and the shape index; equivalent to indirect effect tests of path models).

309 Using 1000 bootstraps of the dataset, we uncovered a significant indirect association between

310 stationary time and shape index $(r=-0.15, p=0.012)$, mediated by their shared association with

311 performance PC 1. This indicated that individuals with more elongate bodies and larger caudal

312 regions tended to exhibit lower stationary time in an open field test owing to the fact that these

313 fish also tended to have higher fast-start performance PC 1 values.

\section{Discussion}

Wild-derived zebrafish selectively bred for divergent stress-coping styles did not merely

317 evolve a set of behavioral differences, but also diverged in body morphology and locomotor 
318 performance. The correlated responses of body shape and fast-start performance to artificial 319 selection on an animal personality trait demonstrates underlying genetic associations among the 320 traits in this study. This means these traits probably cannot evolve independently, as the 321 evolution of one can constrain the evolution of the others. Thus, seemingly unrelated traits like 322 locomotor ability and personality can indeed coevolve. Overall, this study represents one of the 323 earliest documentations of correlations between animal personalities and either whole-body 324 morphology or locomotor performance (Ahlgren, Chapman, Nilsson, \& Brönmark, 2015; 325 Hulthén, Chapman, Nilsson, Hollander, \& Brönmark, 2014; Müller \& von Keyserlingk, 2006). Compared to fish from the shy line, bold fish had enhanced fast-start performance and a 328 phenotype combinations that should provide high fitness in the wild. The two selection lines examined in this study differed by as much as 2.1 standard deviation units in stationary behavior, 330 up to 0.9 standard deviation units in fast-start performance, and exhibited an average Procrustes 331 distance as high as 0.017 . This indicates substantial behavioral divergence, moderate-to-strong 332 divergence in locomotor performance, and moderate shape differences. For comparison, 333 morphological differences between these zebrafish lines were considerably less than observed 334 between populations of Gambusia fishes living with or without predatory fish (Procrustes 335 distances 0.030-0.046; Langerhans, Gifford, \& Joseph, 2007; Langerhans \& Makowicz, 2009;

336 Langerhans, 2009b), but comparable to that seen in the rapid differentiation of a crater lake 337 cichlid, Amphilophus citrinellus (Procrustes distance 0.017; Elmer, Lehtonen, Kautt, Harrod, \& 338 Meyer, 2010). Based on work in other systems, we speculate that the genetic correlations in zebrafish 340 stemmed from correlational selection in the wild via predation (Pruitt, Stachowicz, \& Sih, 2012; 341 Réale \& Festa-Bianchet, 2003). Most natural fish populations are subject to predation, and the 342 limited ecological data on wild zebrafish suggest they regularly encounter avian and fish 343 predators (Bass \& Gerlai, 2008; Engeszer, Patterson, Rao, \& Parichy, 2007; Spence et al., 2006). 344 In the presence of predation, selection might favor both fast-and-bold phenotypes as well as shy345 and-slow phenotypes, leading to correlation in these trait combinations. Bold, exploratory, and 346 risk-taking behaviors might offer a host of fitness advantages only when combined with greater 347 acceleration, maneuverability, and fast-start velocity. Larger caudal regions and increased fast348 start performance can enhance survival during predatory encounters in fish (Langerhans, 2009a; 
Walker, Ghalambor, Griset, McKenney, \& Reznick, 2005). Meanwhile, shy, slow individuals

350 might represent an alternative high-fitness strategy by hiding from predators and experiencing

351 greater longevity (Langerhans, 2006; Smith \& Blumstein, 2008). Moreover, because of the

352 physiological tradeoff between fast-start performance and steady-swimming performance known

353 for many fish, and expected in zebrafish, shy-slow individuals might exhibit higher steady-

354 swimming abilities and thus greater energetic efficiency during routine activities such as

355 foraging and station holding (Langerhans \& Reznick, 2010; Langerhans, 2009b; Oufiero, Walsh,

356 Reznick, \& Garland, 2011; Plaut, 2001).

The responses of morphological and locomotor traits to selection on behavior were

358 particularly strong in female zebrafish: females tended to differ between lines more than males,

359 and in more aspects of fast-start performance (multiple PCs). The cause of this is unclear:

360 perhaps correlational selection in the wild is stronger for females than males (e.g., selection in

361 males may largely favor high fast-start performance regardless of stress coping style), or perhaps

362 genetic and physiological differences between the sexes influence the expression of morphology

363 and performance genes. Male zebrafish in this study and in the wild (Dahlbom, Lagman,

364 Lundstedt-Enkel, Sundström, \& Winberg, 2011, Wong et al., 2012) exhibit greater boldness than

365 females, and male zebrafish also exhibit a more elongate body with a larger caudal region than

366 females. It is possible that high fast-start performance phenotypes are more canalized in males

367 compared to females.

368 The ultimate and proximate causes of genetic associations between personality,

369 morphology, and performance and their prevalence in nature require further study. Our findings

370 not only suggest that correlational selection might ultimately underlie the observed associations,

371 but also that different proximate mechanisms might explain the correlated responses to selection

372 on behavior for the two non-behavioral traits examined here. First, we found that after seven

373 generations in the laboratory, zebrafish exhibited a significant and direct phenotypic correlation

374 between personality (stationary time in an open field test) and locomotor performance (PC 1 of

375 fast-start performance). This is consistent with pleiotropy or physical gene linkage, where either

376 expression of the two traits share some of the same genes or some independent genes that

377 influence the two traits are proximally located on the same chromosome and are thus co-

378 inherited. On the other hand, we found no evidence for a direct phenotypic correlation between

379 personality and body shape after seven generations in the lab, even though body shape had 
diverged between selection lines. Instead, we only observed a significant indirect association between these two traits: personality was indirectly associated with body shape because (1) body shape and fast-start performance showed a strong phenotypic correlation, apparently reflecting biomechanical relationships where body shape partially causes locomotor performance (Domenici, Turesson, Brodersen, \& Brönmark, 2008; Langerhans, 2009a, 2010; Swallow \& Hayes, 2009), and (2) fast-start performance was significantly correlated with personality. These results suggest that the correlated response of body shape to selection on behavior may have reflected linkage disequilibrium between genes that influence morphology and behavior, built up by correlational selection in the wild. Without continued correlational selection, this kind of disequilibrium breaks down quickly under random mating, and is consistent with the lack of direct phenotypic correlation observed in the seventh generation of lab-raised fish in this study.

Although our results strongly suggest that genetic correlations among the traits caused correlated evolutionary responses to artificial selection on one trait, an alternative explanation is possible. That is, correlated responses could reflect socially-induced phenotypic changes, where bold fish that only interact with other bold fish develop different body shapes and locomotor capacities than shy fish that interact only with other shy fish. In this scenario, social interactions, and not underlying genes per se, could create correlated responses to selection (Laskowski \& Pruitt, 2014). Because the different selection lines were housed separately in this study, we cannot rule out this explanation. However, this interpretation seems less robust in light of the evidence for pleiotropy and in the absence of any previously demonstrated socially-induced responses in zebrafish performance or morphology.

Owing to the rich resources available to zebrafish researchers and the ever-increasing use

403 future work in zebrafish that could provide insights into the genetic mechanisms underlying complex trait associations. Zebrafish research has already yielded information on genes and molecular pathways that influence locomotion, hyperactivity, and morphological traits (Granato et al., 1996; Mabee et al., 2007; Norton \& Bally-Cuif, 2010). Interactions among such genes are not well understood. Nor are the interactions among behavioral, physiological, and morphological genes, or the pleiotropic effects of genes across these phenotypic domains. In 409 light of our findings, genotype-phenotype studies focused on behavior-physiology-morphology associations could represent a fruitful area of research. Because correlational selection on animal 
411 personalities and non-behavioral traits might be common in the wild, and because personalities

412 are underlain by mechanisms with broad phenotypic consequences, we might expect personality

413 to often correlate with non-behavioral traits such as morphology and performance (Hulthén et al.,

414 2014; Kim \& Velando, 2015; Lacasse \& Aubin-horth, 2012; Olmos \& Turner, 2008). However,

415 we currently know very little about these associations. If complex behaviors often coevolve with

416 disparate traits like locomotion and morphology, not only will this alter our understanding of

417 whole-organism adaptation and the role of evolutionary constraints among different types of

418 traits, but we might also apply this knowledge to captive and domestic animal breeding, pest

419 management, and understanding associations between human behaviors and other traits (Zilioli,

420 Sell, et al., 2015; Carré \& McCormick, 2008). Results of this study suggest genetic associations

421 exist between personality, morphology, and performance; we now need additional research to

422 understand their frequency and importance.

424 Acknowledgments

425 We thank Ryan Wong and Heather Hill for experimental assistance, Brad Ring and John

426 Davis for animal husbandry, and the members of the Langerhans Lab for comments on the

427 manuscript. This study was supported by the NCSU Initiative for Maximizing Student Diversity

428 to DR (NIH-GM083242), NIH grant (1R21MH080500) to JG, and NSF grant (DEB-0842364) to

429 RBL.

\section{$431 \quad$ References}

Adkins-Regan, E. (2005). Hormones and Animal Social Behavior. Princeton, NJ: Princeton University Press.

Agrawal, A. F., \& Stinchcombe, J. R. (2009). How much do genetic covariances alter the rate of adaptation? Proceedings of the Royal Society B: Biological Sciences, 276(1659), 1183-91. http://doi.org/10.1098/rspb.2008.1671 
Bass, S. L. S., \& Gerlai, R. (2008). Zebrafish (Danio rerio) responds differentially to stimulus fish: the effects of sympatric and allopatric predators and harmless fish. Behavioural Brain Research, 186(1), 107-17. http://doi.org/10.1016/j.bbr.2007.07.037

Biro, P. A., \& Stamps, J. A. (2010). Do consistent individual differences in metabolic rate promote consistent individual differences in behavior? Trends in Ecology \& Evolution, 25, 653-659. http://doi.org/10.1016/j.tree.2010.08.003

Bookstein, F. L. (1996). Biometrics, biomathematics and the morphometric synthesis. Bulletin of Mathematical Biology, 58(2), 313-365. http://doi.org/10.1007/BF02458311

Bourdeau, P. E., \& Johansson, F. (2012). Predator-induced morphological defences as byproducts of prey behaviour: a review and prospectus. Oikos, 121, 1175-1190. http://doi.org/10.1111/j.1600-0706.2012.20235.x

Brodie, E. D. (1989). Genetic correlations between morphology and antipredator behaviour in natural populations of the garter snake Thamnophis ordinoides. Nature, 342(6249), 542-3. http://doi.org/10.1038/342542a0

Brodie, E. D. (1992). Correlational selection for color pattern and antipredator behavior in the garter snake Thamnophis ordinoides. Evolution, 46, 1284-1298. http://doi.org/10.2307/2409937

Careau, V., Réale, D., Humphries, M. M., \& Thomas, D. W. (2010). The pace of life under artificial selection: personality, energy expenditure, and longevity are correlated in domestic dogs. The American Naturalist, 175, 753-758. http://doi.org/10.1086/652435

Carere, C., \& van Oers, K. (2004). Shy and bold great tits (Parus major): body temperature and breath rate in response to handling stress. Physiology \& Behavior, 82, 905-912. http://doi.org/10.1016/j.physbeh.2004.07.009

Cheverud, J. M. (1984). Quantitative genetics and developmental constraints on evolution by selection. Journal of Theoretical Biology, 110, 155-171. http://doi.org/10.1016/S00225193(84)80050-8

Cohen, J. (1988). Statistical Power Analysis for the Behavioral Sciences. Hillsdale, NJ: Erlbaum.

Dahlbom, S. J., Lagman, D., Lundstedt-Enkel, K., Sundström, L. F., \& Winberg, S. (2011). Boldness predicts social status in zebrafish (Danio rerio). PloS One, 6(8), e23565. http://doi.org/10.1371/journal.pone.0023565

Dewitt, T. J., Sih, A., \& Hucko, J. A. (1999). Trait compensation and cospecialization in a freshwater snail: size, shape and antipredator behaviour. Animal Behaviour, 58, 397-407. http://doi.org/10.1006/anbe.1999.1158 
Dickey, C. C., McCarley, R. W., \& Shenton, M. E. (2002). The brain in schizotypal personality disorder: a review of structural MRI and CT findings. Harvard Review of Psychiatry, 10, 115.

Dill, L. M. (1974). The escape response of the zebra danio (Brachydanio rerio) I. The stimulus for escape. Animal Behaviour, 22(3), 711-722. http://doi.org/10.1016/S00033472(74)80022-9

Domenici, P., Turesson, H., Brodersen, J., \& Brönmark, C. (2008). Predator-induced morphology enhances escape locomotion in crucian carp. Proceedings of the Royal Society B: Biological Sciences, 275, 195-201. http://doi.org/10.1098/rspb.2007.1088

Elmer, K. R., Lehtonen, T. K., Kautt, A. F., Harrod, C., \& Meyer, A. (2010). Rapid sympatric ecological differentiation of crater lake cichlid fishes within historic times. BMC Biology, 8(1), 60. http://doi.org/10.1186/1741-7007-8-60

Endler, J. A. (1995). Multiple-trait coevolution and environmental gradients in guppies. Trends in Ecology and Evolution, 10, 22-29. http://doi.org/10.1016/S0169-5347(00)88956-9

Engeszer, R. E., Patterson, L. B., Rao, A. A., \& Parichy, D. M. (2007). Zebrafish in the wild: a review of natural history and new notes from the field. Zebrafish, 4, 21-40. http://doi.org/10.1089/zeb.2006.9997

Falconer, D., \& MacKay, T. (1996). Introduction to Quantitative Genetics (4th ed.). Burnt Mill, Harlow, United Kingdom: Longman Scientific \& Technical.

Ghalambor, C. K., Walker, J. A., \& Reznick, D. N. (2003). Multi-trait selection, adaptation, and constraints on the evolution of burst swimming performance. Integrative and Comparative Biology, 43(3), 431-438. http://doi.org/10.1093/icb/43.3.431

Granato, M., van Eeden, F. J., Schach, U., Trowe, T., Brand, M., Furutani-Seiki, M., ... Nusslein-Volhard, C. (1996). Genes controlling and mediating locomotion behavior of the zebrafish embryo and larva. Development, 123(1), 399-413.

Houde, A. E. (1994). Effect of artificial selection on male colour patterns on mating preference of female guppies. Proceedings of the Royal Society B: Biological Sciences. http://doi.org/10.1098/rspb.1994.0059

Hulthén, K., Chapman, B. B., Nilsson, P. A., Hollander, J., \& Brönmark, C. (2014). Express yourself: bold individuals induce enhanced morphological defences. Proceedings of the Royal Society B: Biological Sciences, 281(1776), 20132703. http://doi.org/10.1098/rspb.2013.2703

Johansson, F., \& Andersson, J. (2009). Scared fish get lazy, and lazy fish get fat. The Journal of Animal Ecology, 78, 772-777. http://doi.org/10.1111/j.1365-2656.2009.01530.x 
Jones, A. G., Arnold, S. J., \& Bürger, R. (2003). Stability of the G-matrix in a population experiencing pleiotropic mutation, stabilizing selection, and genetic drift. Evolution, 57(8), 1747-1760. http://doi.org/10.1111/j.0014-3820.2003.tb00583.x

Ketterson, E. D., Atwell, J. W., \& McGlothlin, J. W. (2009). Phenotypic integration and independence: hormones, performance, and response to environmental change. Integrative and Comparative Biology, 49(4), 365-379. http://doi.org/10.1093/icb/icp057

Kim, S.-Y., \& Velando, A. (2015). Phenotypic integration between antipredator behavior and camouflage pattern in juvenile sticklebacks. Evolution, 69(3), 830-8. http://doi.org/10.1111/evo.12600

Koolhaas, J. M., de Boer, S. F., Coppens, C. M., \& Buwalda, B. (2010). Neuroendocrinology of coping styles: towards understanding the biology of individual variation. Frontiers in Neuroendocrinology, 31(3), 307-21. http://doi.org/10.1016/j.yfrne.2010.04.001

Lacasse, J., \& Aubin-horth, N. (2012). A test of the coupling of predator defense morphology and behavior variation in two threespine stickleback populations. Current Zoology, 58, 5365 .

Lande, R. (1979). Quantitative genetic analysis of multivariate allometry, applied to brain: body size allometry. Evolution, 33(1), 402-416.

Lande, R., \& Arnold, S. J. (1983). The measurement of selection on correlated characters. Evolution, 37(6), 1210-1226.

Langerhans, R. B. (2006). Evolutionary consequences of predation: avoidance, escape, reproduction, and diversification. In A. M. T. Elewa (Ed.), Predation in Organisms: a Distinct Phenomenon (pp. 177-220). Heidelberg, Germany: Springer-Verlag.

Langerhans, R. B. (2009a). Morphology, performance, fitness: functional insight into a postPleistocene radiation of mosquitofish. Biology Letters, 5, 488-491. http://doi.org/10.1098/rsbl.2009.0179

Langerhans, R. B. (2009b). Trade-off between steady and unsteady swimming underlies predator-driven divergence in Gambusia affinis. Journal of Evolutionary Biology, 22b, 1057-1075. http://doi.org/10.1111/j.1420-9101.2009.01716.x

Langerhans, R. B. (2010). Predicting evolution with generalized models of divergent selection: a case study with poeciliid fish. Integrative and Comparative Biology, 50, 1167-1184. http://doi.org/10.1093/icb/icq117

Langerhans, R. B., Gifford, M. E., \& Joseph, E. O. (2007). Ecological speciation in Gambusia fishes. Evolution, 61(9), 2056-74. http://doi.org/10.1111/j.1558-5646.2007.00171.x 
Langerhans, R. B., \& Makowicz, A. M. (2009). Shared and unique features of morphological differentiation between predator regimes in Gambusia caymanensis. Journal of Evolutionary Biology, 22(11), 2231-42.

Langerhans, R. B., \& Reznick, D. N. (2010). Ecology and evolution of swimming performance in fishes: predicting evolution with biomechanics. In P. Domenici \& B. G. Kapoor (Eds.), Fish Locomotion: an Eco-ethological Perspective (pp. 200-248). Enfield, NH: Science Publishers.

Laskowski, K. L., \& Pruitt, J. N. (2014). Evidence of social niche construction: persistent and repeated social interactions generate stronger personalities in a social spider. Proceedings of the Royal Society B: Biological Sciences, 281(1783), 20133166. http://doi.org/10.1098/rspb.2013.3166

Losos, J. B., Creer, D. A., \& Schulte II, J. A. (2002). Cautionary comments on the measurement of maximum locomotor capabilities. Journal of Zoology, 258(1), 57-61. http://doi.org/10.1017/S0952836902001206

Lynch, M., \& Walsh, J. B. (1998). Genetics and Analysis of Quantitative Traits. Sunderland, MA: Sinauer.

Mabee, P. M., Arratia, G., Coburn, M., Haendel, M., Hilton, E. J., Lundberg, J. G., ... Westerfield, M. (2007). Connecting evolutionary morphology to genomics using ontologies: a case study from Cypriniformes including zebrafish. Journal of Experimental Zoology. Part B, Molecular and Developmental Evolution, 308(5), 655-68. http://doi.org/10.1002/jez.b.21181

McGlothlin, J. W., \& Ketterson, E. D. (2008). Hormone-mediated suites as adaptations and evolutionary constraints. Philosophical Transactions of the Royal Society of London. Series B, Biological Sciences, 363(1497), 1611-20. http://doi.org/10.1098/rstb.2007.0002

McGlothlin, J. W., Parker, P. G., Nolan, V., \& Ketterson, E. D. (2005). Correlational selection leads to genetic integration of body size and an attractive plumage trait in dark-eyed juncos. Evolution, 59, 658-671. http://doi.org/10.1111/j.0014-3820.2005.tb01024.x

Müller, R., \& von Keyserlingk, M. A. G. (2006). Consistency of flight speed and its correlation to productivity and to personality in Bos taurus beef cattle. Applied Animal Behaviour Science, 99(3), 193-204.

Murren, C. J. (2012). The integrated phenotype. Integrative and Comparative Biology, 52(1), 64-76. http://doi.org/10.1093/icb/ics043

Norton, W., \& Bally-Cuif, L. (2010). Adult zebrafish as a model organism for behavioural genetics. BMC Neuroscience, 11(1), 90. http://doi.org/10.1186/1471-2202-11-90 
Olmos, G., \& Turner, S. P. (2008). The relationships between temperament during routine handling tasks, weight gain and facial hair whorl position in frequently handled beef cattle. Applied Animal Behaviour Science, 115, 25-36. http://doi.org/10.1016/j.applanim.2008.05.001

Oufiero, C. E., Walsh, M. R., Reznick, D. N., \& Garland, T. (2011). Swimming performance trade-offs across a gradient in community composition in Trinidadian killifish (Rivulus hartii). Ecology, 92(1), 170-9.

Øverli, Ø., Sørensen, C., Pulman, K. G. T., Pottinger, T. G., Korzan, W., Summers, C. H., \& Nilsson, G. E. (2007). Evolutionary background for stress-coping styles: relationships between physiological, behavioral, and cognitive traits in non-mammalian vertebrates. Neuroscience and Biobehavioral Reviews, 31(3), 396-412. http://doi.org/10.1016/j.neubiorev.2006.10.006

Phillips, P. C., \& Arnold, S. J. (1989). Visualizing multivariate selection. Evolution, 43, 12091222. http://doi.org/10.2307/2409357

Pigliucci, M., \& Preston, K. A. (2004). Phenotypic Integration. Oxford: Oxford University Press.

Plaut, I. (2001). Critical swimming speed: its ecological relevance. Comparative Biochemistry and Physiology Part A: Molecular \& Integrative Physiology, 131(1), 41-50. http://doi.org/10.1016/S1095-6433(01)00462-7

Pruitt, J. N., \& Riechert, S. E. (2012). The ecological consequences of temperament in spiders. Current Zoology, 58(4), 589-596.

Pruitt, J. N., Stachowicz, J. J., \& Sih, A. (2012). Behavioral types of predator and prey jointly determine prey survival: potential implications for the maintenance of within-species behavioral variation. The American Naturalist, 179(2), 217-227. http://doi.org/10.1086/663680

Réale, D., Dingemanse, N. J., Kazem, A. J. N., \& Wright, J. (2010). Evolutionary and ecological approaches to the study of personality. Philosophical Transactions of the Royal Society of London. Series B, Biological Sciences, 365, 3937-3946. http://doi.org/10.1098/rstb.2010.0222

Réale, D., \& Festa-Bianchet, M. (2003). Predator-induced natural selection on temperament in bighorn ewes. Animal Behaviour, 65(3), 463-470. http://doi.org/10.1006/anbe.2003.2100

Réale, D., Garant, D., Humphries, M. M., Bergeron, P., Careau, V., \& Montiglio, P.-O. (2010). Personality and the emergence of the pace-of-life syndrome concept at the population level. Philosophical Transactions of the Royal Society of London. Series B, Biological Sciences, 365, 4051-4063. http://doi.org/10.1098/rstb.2010.0208 
Réale, D., Reader, S. M., Sol, D., McDougall, P. T., \& Dingemanse, N. J. (2007). Integrating animal temperament within ecology and evolution. Biological Reviews of the Cambridge Philosophical Society, 82, 291-318. http://doi.org/10.1111/j.1469-185X.2007.00010.x

Ribas, L., \& Piferrer, F. (2014). The zebrafish (Danio rerio) as a model organism, with emphasis on applications for finfish aquaculture research. Reviews in Aquaculture, 6(4), 209-240. http://doi.org/10.1111/raq.12041

Robison, B. D., \& Rowland, W. (2005). A potential model system for studying the genetics of domestication: behavioral variation among wild and domesticated strains of zebra danio (Danio rerio). Canadian Journal of Fisheries and Aquatic Sciences, 62(9), 2046-2054. http://doi.org/10.1139/f05-118

Roff, D. A., \& Fairbairn, D. J. (2012). A test of the hypothesis that correlational selection generates genetic correlations. Evolution, 66(9), 2953-60. http://doi.org/10.1111/j.15585646.2012.01656.x

Rohlf, F. J. (2003). TpsSmall, v. 1.20. Stony Brook, NY: State University of New York.

Rohlf, F. J. (2010). TpsRelw. Stony Brook, NY: State University of New York.

Rohlf, F. J. (2012). TpsUtil. Stony Brook, NY: State University of New York.

Rohlf, F. J. (2013). TpsDig. Stony Brook, NY: State University of New York.

Ruzicka, L., Bradford, Y. M., Frazer, K., Howe, D. G., Paddock, H., Ramachandran, S., ... Westerfield, M. (2015). ZFIN, The zebrafish model organism database: updates and new directions. Genesis, 53(8), 498-509. http://doi.org/10.1002/dvg.22868

Santos, J. C., \& Cannatella, D. C. (2011). Phenotypic integration emerges from aposematism and scale in poison frogs. Proceedings of the National Academy of Sciences of the United States of America, 108(15), 6175-80. http://doi.org/10.1073/pnas.1010952108

Schluter, D. (1996). Ecological causes of adaptive radiation. The American Naturalist, 148, S40. http://doi.org/10.1086/285901

Schluter, D. (2010). Resource competition and coevolution in sticklebacks. Evolution: Education and Outreach. http://doi.org/10.1007/s12052-009-0204-6

Selman, C., Lumsden, S., Bünger, L., Hill, W. G., \& Speakman, J. R. (2001). Resting metabolic rate and morphology in mice (Mus musculus) selected for high and low food intake. The Journal of Experimental Biology, 204, 777-784.

Sih, A., Bell, A., \& Johnson, J. C. (2004). Behavioral syndromes: an ecological and evolutionary overview. Trends in Ecology and Evolution, 19, 372-378.

http://doi.org/10.1016/j.tree.2004.04.009 
Sih, A., Cote, J., Evans, M., Fogarty, S., \& Pruitt, J. (2012). Ecological implications of behavioural syndromes. Ecology Letters, 15(3), 278-289. http://doi.org/10.1111/j.14610248.2011.01731.x

Sinervo, B., \& Svensson, E. (2002). Correlational selection and the evolution of genomic architecture. Heredity, 89(5), 329-38. http://doi.org/10.1038/sj.hdy.6800148

Smith, B. R., \& Blumstein, D. T. (2008). Fitness consequences of personality: a meta-analysis. Behavioral Ecology, 19, 448-455. http://doi.org/10.1093/beheco/arm144

Spence, R., Fatema, M. K., Reichard, M., Huq, K. A., Wahab, M. A., Ahmed, Z. F., \& Smith, C. (2006). The distribution and habitat preferences of the zebrafish in Bangladesh. Journal of Fish Biology, 69(5), 1435-1448. http://doi.org/10.1111/j.1095-8649.2006.01206.x

Swallow, J., \& Hayes, J. (2009). Selection experiments and experimental evolution of performance and physiology. In T. Garland Jr \& M. R. Rose (Eds.), Experimental Evolution: Concepts, Methods, and Applications of Selection Experiments (pp. 301-351). Los Angeles: University of California.

Trut, L. N., Oskina, I., \& Kharlamova, A. (2009). Animal evolution during domestication: the domesticated fox as a model. BioEssays $\square$ : News and Reviews in Molecular, Cellular and Developmental Biology, 31(3), 349-60. http://doi.org/10.1002/bies.200800070

Trut, L. N., Plyusnina, I. Z., \& Oskina, I. N. (2004). An experiment on fox domestication and debatable issues of evolution of the dog. Russian Journal of Genetics, 40(6), 644-655. http://doi.org/10.1023/B:RUGE.0000033312.92773.c1

Tytell, E. D., \& Lauder, G. V. (2008). Hydrodynamics of the escape response in bluegill sunfish, Lepomis macrochirus. The Journal of Experimental Biology, 211(Pt 21), 3359-69. http://doi.org/10.1242/jeb.020917

Vervust, B., Grbac, I., \& Van Damme, R. (2007). Differences in morphology, performance and behaviour between recently diverged populations of Podarcis sicula mirror differences in predation pressure. Oikos, 116, 1343-1352. http://doi.org/10.1111/j.2007.00301299.15989.x

Walker, J. A. (1998). Estimating velocities and accelerations of animal locomotion: a simulation experiment comparing numerical differentiation algorithms. Journal of Experimental Biology, 201, 981-995. http://doi.org/10.1002/pds.1118

Walker, J. A., Ghalambor, C. K., Griset, O. L., McKenney, D., \& Reznick, D. N. (2005). Do faster starts increase the probability of evading predators? Functional Ecology, 19(5), 808815. http://doi.org/10.1111/j.1365-2435.2005.01033.x 
674 Wilkinson, G. S., \& Reillo, P. R. (1994). Female choice response to artificial selection on an 675 exaggerated male trait in a stalk-eyed fly. Proceedings of the Royal Society B: Biological $676 \quad$ Sciences, 255, 1-6. http://doi.org/10.1098/rspb.1994.0001

677 Wolf, M., \& Weissing, F. J. (2012). Animal personalities: consequences for ecology and $678 \quad$ evolution. Trends in Ecology \& Evolution, 27(8), 452-461.

679 Wolf, M., \& Werner, Y. L. (1994). The striped colour pattern and striped/non-striped 680 polymorphism in snakes (Reptilia: Ophidia). Biological Reviews, 69(4), 599-610. $681 \quad$ http://doi.org/10.1111/j.1469-185X.1994.tb01250.x

682 Wong, R. Y., Perrin, F., Oxendine, S. E., Kezios, Z. D., Sawyer, S., Zhou, L., ... Godwin, J. 683 (2012). Comparing behavioral responses across multiple assays of stress and anxiety in zebrafish (Danio rerio). Behaviour, 149(10-12), 1205-1240. http://doi.org/10.1163/1568539X-00003018

Wright, D., Nakamichi, R., Krause, J., \& Butlin, R. K. (2006). QTL analysis of behavioral and morphological differentiation between wild and laboratory zebrafish (Danio rerio).

689 Zelditch, M. L., Swiderski, D. L., \& Sheets, H. D. (2012). Geometric Morphometrics for Biologists: a Primer. Waltham, MA: Academic Press.

691

692 


\section{$693 \quad$ Figure Legends}

694 Figure 1. Landmarks used for morphological analysis (a), and morphological differences

695 between bold and shy phenotype lines of zebrafish females $(b)$ and males $(c)$. Body shape

696 variation along the shape index (see text) depicted with thin-plate spline transformation grids (no

697 magnification; solid lines connecting outer landmarks are drawn to aid interpretation). Landmark

698 vectors beneath each set of grids convey the direction and relative magnitude of change in the

699 location of each landmark, pointing toward values characteristic of bold lines.

701 Figure 2. Variation in body morphology and fast-start performance (captured by PC 1) between

702 bold (filled symbols) and shy (open symbols) coping style lines in female (squares) and male 703 (circles) zebrafish.

704 
705 Table 1. PC loadings for kinematic variables used to measure locomotor performance. PC 1

706 captured most of the variation in velocity and maximum acceleration, PC 2 largely described

707 variation in turning angle, and PC 3 partially captured average acceleration.

\begin{tabular}{lccc} 
Performance variable & PC 1 & PC 2 & PC 3 \\
\hline Maximum velocity & $\mathbf{0 . 9 3}$ & -0.13 & -0.15 \\
Maximum acceleration & $\mathbf{0 . 6 7}$ & -0.08 & -0.59 \\
Average velocity & $\mathbf{0 . 7 9}$ & -0.18 & 0.29 \\
Average acceleration & 0.06 & -0.57 & $\mathbf{0 . 6 4}$ \\
Turning angle & 0.11 & $\mathbf{0 . 8 3}$ & 0.29 \\
Rotational velocity & $\mathbf{0 . 7 5}$ & 0.35 & 0.31 \\
\hline Variance explained: & $41.84 \%$ & $19.85 \%$ & $17.59 \%$
\end{tabular}

708

709 
710 Table 2. Results of MANCOVA examining how body shape varies with coping style line

711 (bold/shy), sex, and size.

\begin{tabular}{lccc} 
Source & $\boldsymbol{F}$ & D.f. & $\boldsymbol{P}$ \\
\hline Coping style line & 2.37 & 11,108 & 0.0115 \\
Sex & 7.80 & 11,108 & $<0.0001$ \\
Sex $\times$ coping style line & 0.73 & 11,108 & 0.7052 \\
Centroid size & 4.88 & 11,108 & $<0.0001$ \\
Generation & 3.66 & 22,216 & $<0.0001$ \\
\hline
\end{tabular}

712

713

714

715

716 Table 3. Results of general linear models examining variation in swimming performance

717 (captured by PCs) between bold and shy zebrafish lines.

\begin{tabular}{|c|c|c|c|c|c|c|c|}
\hline \multirow[b]{2}{*}{ Source } & \multirow[b]{2}{*}{ D.f. } & \multicolumn{2}{|c|}{ PC 1} & \multicolumn{2}{|c|}{ PC 2} & \multicolumn{2}{|c|}{ PC 3} \\
\hline & & $\boldsymbol{F}$ & $P$ & $\boldsymbol{F}$ & $\boldsymbol{P}$ & $F$ & $P$ \\
\hline Coping style line & 1,118 & 10.88 & 0.0013 & 0.08 & 0.7716 & 1.05 & 0.3078 \\
\hline Sex & 1,118 & 4.94 & 0.0281 & 1.28 & 0.2608 & 1.54 & 0.2174 \\
\hline \multicolumn{8}{|l|}{ Sex $\times$ coping style } \\
\hline line & 1,118 & 4.05 & 0.0464 & 3.19 & 0.0768 & 6.32 & 0.0133 \\
\hline Centroid size & 1,118 & 4.76 & 0.0310 & 0.09 & 0.7588 & 0.03 & 0.8610 \\
\hline Generation & 2,118 & 11.46 & $<0.0001$ & 1.37 & 0.2571 & 25.93 & $<0.0001$ \\
\hline
\end{tabular}

718 
Figure 1

(a)

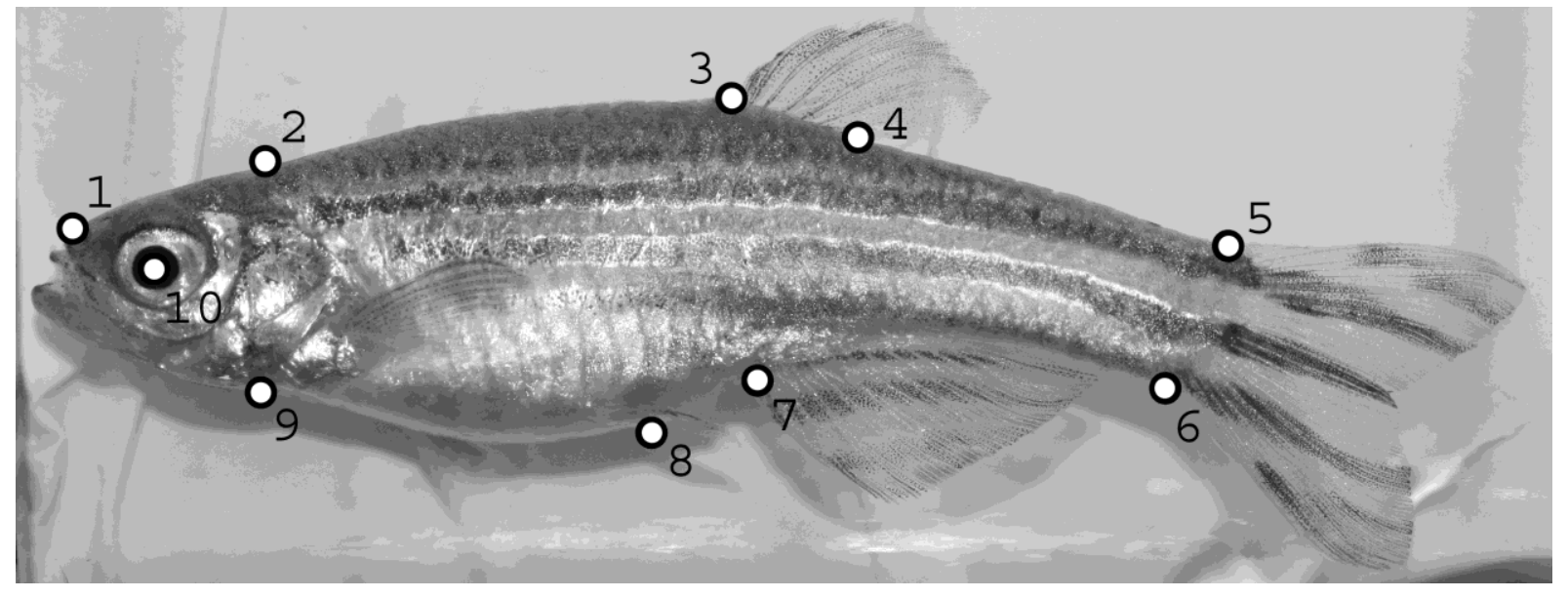

(b)

Shy

Bold

Females
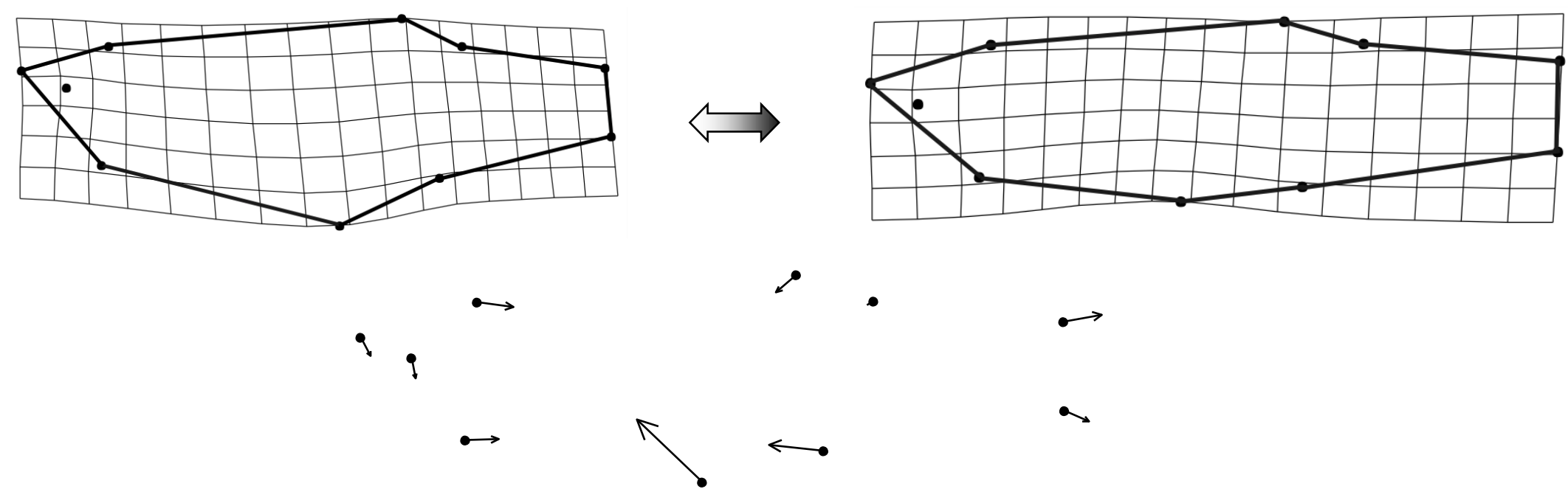

(c)

Males
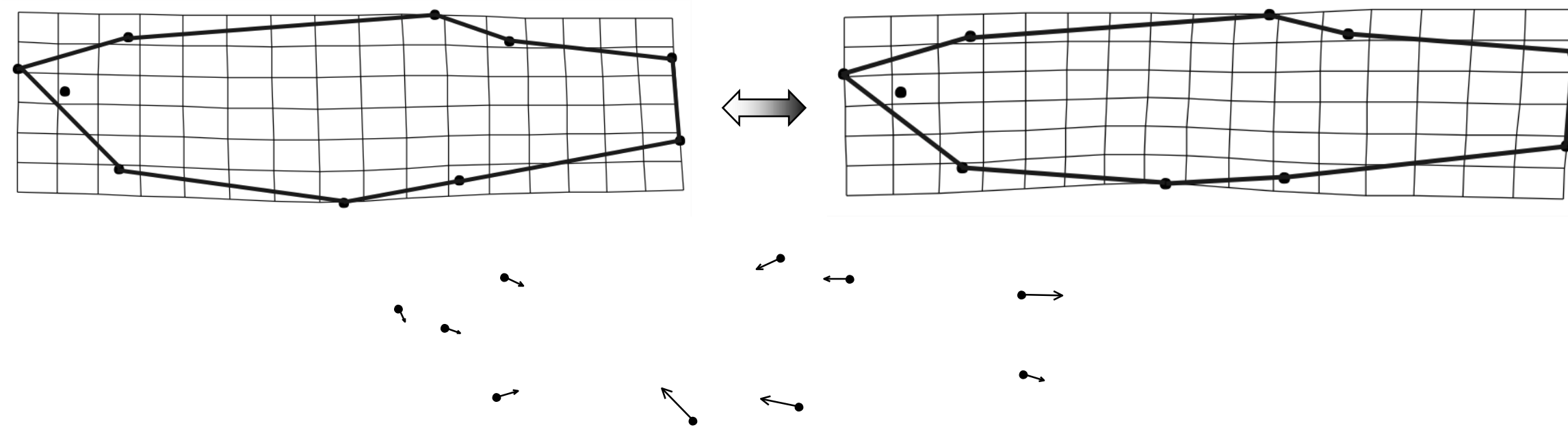
Figure 2

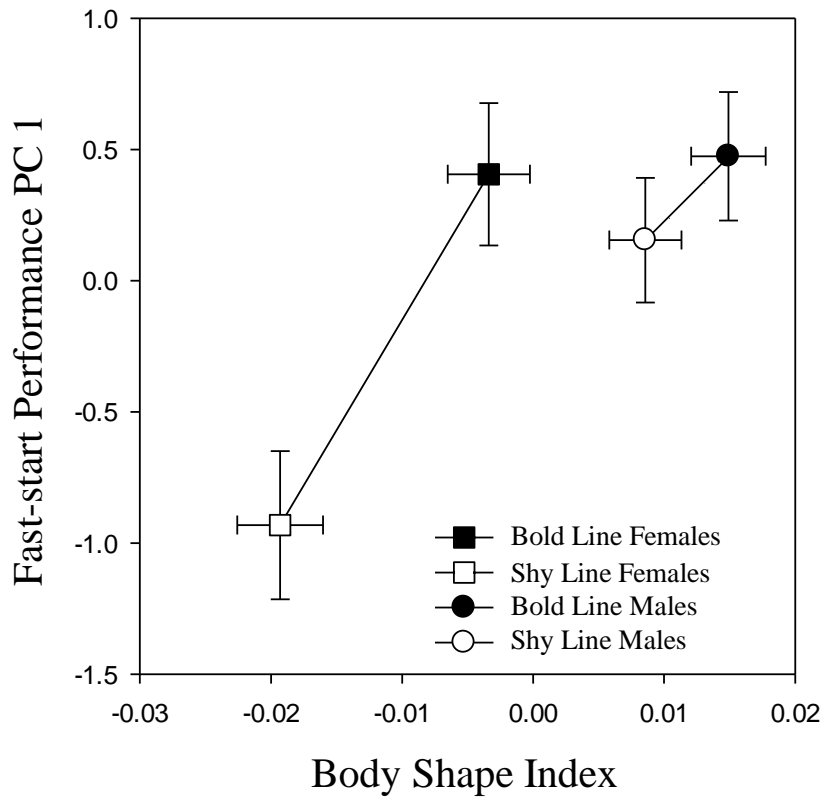

